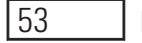

B-TYPE NATRIURETIC PEPTIDE PERFORMS BETTER THAN CURRENT CARDIOVASCULAR RISK SCORES IN IDENTIFYING SILENT "PANCARDIAC" TARGET ORGAN DAMAGE IN ALREADY TREATED PRIMARY PREVENTION PATIENTS

doi:10.1136/heartjnl-2011-300198.53

${ }^{1}$ A Nadir, 'S Rekhraj, ${ }^{2} \mathrm{~J}$ Davidson, ${ }^{1} \mathrm{~T}$ M MacDonald, ${ }^{1} \mathrm{C}$ C Lang, ${ }^{1}$ A D Struthers. ${ }^{1}$ University of Dundee, Dundee, UK; ${ }^{2}$ Ninewells Hospital, Dundee

Background Primary prevention needs to be improved because up to $70 \%$ of cardiovascular (CV) events occur outwith those classified as high risk by CV risk scores currently used in clinical practice (eg, Framingham). One possible way to improve primary prevention of $\mathrm{CV}$ disease is to identify those patients who may already harbour silent pancardiac target organ damage in the form of left ventricular hypertrophy (LVH), systolic dysfunction (LVSD), diastolic dysfunction (LVDD), left atrial enlargement (LAE) or silent myocardial ischaemia. This could be achieved by reapplying traditional CV risk scores to primary prevention patients after they have been treated or by screening with a simple biomarker like B-type natriuretic peptide (BNP).

Methods We prospectively recruited 300 asymptomatic individuals without known cardiovascular disease already on primary prevention therapy. Patients with valvular heart disease, atrial fibrillation and renal impairment were excluded. We measured BNP and calculated 10year global CV risk scores (based on Framingham, ORISK and ASSIGN) in each participant. Transthoracic echocardiography was used to assess LV mass, LV systolic and diastolic function, and left atrial volume while the presence of inducible ischaemia was assessed by dobutamine stress echocardiography or dipyridamole myocardial perfusion imaging. Patients were divided into low, intermediate and high risk groups based on 10-year global CV risk. The prevalence of various cardiac TOD in each group was compared and ROC curves were constructed for BNP and for 10-year global CV risk scores to assess their ability to detect presence of silent cardiac TOD.

Results One hundred and two (34\%) patients (Mean age $64 \pm 6.0$ years, $58 \%$ males) had evidence of silent cardiac TOD (29.7\% LVH, 18\% LAE, $17.3 \%$ LVDD, $7.3 \%$ LVSD and $6.3 \%$ Ischaemia). The prevalence of cardiac TOD ranged from 19 to $28 \%$ in the low risk, $26 \%-33 \%$ in the intermediate risk and $36 \%-41 \%$ in the high risk groups based on three commonly used CV risk equations. BNP levels were significantly higher (median (IOR); 21.6 $(13.6-40.0)$ vs $11.4(6.3-20.0) \mathrm{pg} / \mathrm{ml}, \mathrm{p}<0.0001)$ in those with cardiac TOD compared to those without. The AUC for BNP to identify any form of cardiac TOD was $0.77(p<0.0001)$ overall and $0.83(p<0.0001)$ in males. However, discrimination power of CV risk scores was poor with area under curve of only $0.58(p=0.02)$ for ORISK, $0.62(p=0.001)$ for Framingham and $0.62(p=0.001)$ for ASSIGN to detect presence of any form of TOD.

Conclusion Silent cardiac TOD is highly prevalent (34\%) in already treated primary prevention population but current CV risk estimation alone performs poorly in the detection of these silent cardiac abnormalities. However, a raised BNP is able to identify existing silent cardiac TOD of various subtypes particularly in males. Using BNP to identify silent cardiac TOD could, in the future, become a new way to improve the primary prevention of CV events.

54 CAN MICROALBUMINURIA IDENTIFY SILENT "PANCARDIAC" TARGET ORGAN DAMAGE IN A NON-DIABETIC PRIMARY PREVENTION POPULATION?

doi:10.1136/heartjnl-2011-300198.54

${ }^{1}$ A Nadir, 'S Rekhraj, ${ }^{2} J$ Davidson, ${ }^{1} \mathrm{~T}$ M MacDonald, ${ }^{1} \mathrm{C}$ C Lang, ${ }^{1}$ A D Struthers. ${ }^{1}$ University of Dundee, Dundee, UK; ${ }^{2}$ Ninewells Hospital, Dundee, UK

Background Microalbuminuria is associated with increased cardiovascular mortality and is a marker of generalised vascular dysfunc- tion. We sought to investigate whether micoalbuminuria identified the presence of silent "pancardiac" target organ damage (TOD) ie, left ventricular hypertrophy (LVH), systolic dysfunction (LVSD), diastolic dysfunction (LVDD), left atrial enlargement (LAE) or silent myocardial ischaemia in a non-diabetic primary prevention population.

Methods Two hundred and sixty-three asymptomatic individuals without diabetes or previous cardiovascular disease on primary preventive therapy were prospectively recruited. Each participant underwent a comprehensive echocardiographic examination for the assessment of LV mass, LV systolic and diastolic function, and left atrial volume while the presence of inducible ischaemia was assessed by dobutamine stress echocardiography or dipyridamole myocardial perfusion imaging. A spot urine sample was analysed for microalbuminuria and urinary creatinine by a trained laboratory technician blinded to clinical or echocardiographic data. Microalbuminuria was defined as urinary albumin to creatinine ratio of $\geq 2.5 \mathrm{mg} / \mathrm{mmol}$ in males and $\geq 3.5 \mathrm{mg} / \mathrm{mmol}$ in females.

Results Out of 263 participants (Mean age 64 66.3 years, $57 \%$ males) 89 (33.8\%) had evidence of silent cardiac TOD $(29.7 \% \mathrm{LVH}$, 16.7\% LAE, 17.1\% LVDD, 6.8\% LVSD and 6.1\% Ischaemia). The prevalence of cardiac TOD was significantly higher $53 \%$ vs $29 \%$, $\mathrm{p}=0.002$ ) among those with microalbuminuria than those without. In multivariate analysis adjusted for age, gender, hypertension and dyslipidemia, presence of microalbuminuria was an independent predictor of cardiac TOD with an adjusted HR of 2.54 (95\% CI, 1.2 to $4.4, p=0.005)$. The utility of UACR in discriminating between those with or without cardiac TOD was assessed by receiver operating characteristic analysis but the area under curve was only $0.61 \pm 0.04, p=0.003$.

Conclusion Microalbuminuria is an independent predictor of silent "pancardiac" target organ damage in a non-diabetic primary prevention population. Presence of microalbuminuria may help to identify those primary prevention patients who are at a particularly higher risk.

\section{GENE-GENE INTERACTIONS IN CORONARY ARTERY DISEASE}

doi:10.1136/heartjnl-2011-300198.55

${ }^{1} \mathrm{M} D$ Musameh, ${ }^{2} \mathrm{~W}$ Y S Wang, ${ }^{3} \mathrm{~A}$ J Balmforth, ${ }^{4} \mathrm{~S}$ G Ball, ${ }^{5} \mathrm{~A}$ S Hall, ${ }^{1} \mathrm{M}$ Tomaszewski, ${ }^{1} \mathrm{~N} J$ Samani. ${ }^{1}$ Department of Cardiovascular Sciences, University of Leicester, Glenfield Hospital; Leicester BRU, Leicester, UK; ${ }^{2}$ School of Medical Sciences and Institute for Biomedical Research, University of Sydney, Sydney, Australia; ${ }^{3}$ Division of Cardiovascular and Diabetes Research, University of Leeds, Leeds, UK; ${ }^{4}$ LIGHT Research Institute, Faculty of Medicine and Health, University of Leeds, Leeds, UK; ${ }^{5}$ Division of Cardiovascular and Neuronal Remodelling, University of Leeds, Leeds, UK

Background Only a small fraction of the heritability of coronary artery disease (CAD) has been explained by common variants identified by genome-wide association studies. Among the stones to be turned in the hunt for the missing heritability of CAD are genegene interactions. We investigated whether interactions between common alleles in genes and pathways of known importance to cardiovascular regulation may contribute to the heritability of $\mathrm{CAD}$. Methods 2101 CAD cases and 2426 controls of Caucasian origin recruited into Wellcome Trust Case Control Consortium were genotyped using $50 \mathrm{~K} \mathrm{IBC}$ gene-centric array containing 45707 single nucleotide polymorphisms (SNPs) of the highest biological relevance to cardiovascular system. After applying appropriate quality control filters, 11332 common (minor allele frequency $>10 \%$ ), independent $\left(\mathrm{r}^{2}\right.$ linkage disequilibrium coefficient of $\left.\leq 0.5\right)$ were included in pair-wise SNP-SNP interaction analysis using two complementary statistical approaches: logistic regression (PLINK and INTERSNP software packages) and Bayesian model (BEAM software).

Results None of the analysed SNP-SNP interactions was statistically significant after correction for multiple testing $\left(p=7.8 \times 10^{-10}\right)$. The most significant interaction identified in this analysis was 